\title{
High Impedance Fault and Heavy Load under Big Data Context
}

\author{
Chun Sing Lai, Student Member, IEEE \\ Energy and Power Group \\ University of Oxford \\ Oxfordshire, UK \\ chun.lai@eng.ox.ac.uk
}

\begin{abstract}
Detecting and identifying High Impedance Fault (HIF) in distribution system is an essential task for a secure and reliable grid. The ability for relaying system to differentiate HIF and heavy load condition is still a challenging problem as their current and voltage characteristics are very similar. This paper demonstrates how discrete wavelet transform can be used to identify the two cases. It also reviews how big data analytics could be used to improve this long standing problem. A simple power system model constructed in DIgSILENT PowerFactory performs the short circuit analysis for the HIF and the open circuit islanding for the heavy load conditions. MATLAB will be used for discrete wavelet transform analysis for the output from the power system model.
\end{abstract}

Index Terms-High Impedance Fault, Heavy Load, Big Data, Grid Analytics

\section{INTRODUCTION}

High-impedance faults (HIFs) are defined as unwanted electrical contact between energized conductors and nonconducting materials. The most common type of high impedance faults are caused due to a live wire is touching with trees, concrete walls, or ground. In general, distribution feeder fault detection schemes monitor only low impedance faults and large increments of current due to a low impedance path to ground (short-circuit). Therefore, it is difficult to detect such faults with the methods because it presents only a small increment in line current and this appears to be a normal load increment. If no alert or action is taken, this will be a risk to human life. Most high impedance faults can be detected using harmonic current phase-angle analysis and localized by using recloser-sectionalizer technology [1-3].

The fact that the transient signal during a fault or load switching is non-stationary, containing fundamental frequency component, DC offset with damping, harmonics is one of the problems associated with the traditional protection schemes. In some severe situations, it will cause an inaccurate estimation of phasor representation of the faulted signal, which may cause mal-operation in relays. When this happens the thresholds may have to be calculated again, which may not be useful in real-time situations [4].

\section{REVIEW OF SOLUTIONS TO HIGH IMPEDANCE FAULT}

Since the beginning of 70's, HIF has been studied and several algorithms and techniques were proposed for the detection of such faults, including the decision trees (DTs) and harmonic content, using Least Error Squares Technique, application of wavelets, etc. [5][6].

Distance relay calculates impedance using voltage and current measured at busbars and terminal and detects a fault in case the calculated value converges into the protective zone.

In general, distance relay have tolerance up to $120 \%$ of line impedance between substation and sectioning post before being triggered.

$$
Z_{F I}=\frac{V_{T}}{\left(I_{1}+I_{2}\right) / 2}
$$

$Z_{F I}$ is the fault impedance;

$V_{T}$ is the voltage between Terminal and Single Busbar

$I_{1}$ and $I_{2}$ are the current of the Terminal and Single Busbar respectively

$\Delta I$ fault selective relay current compares the magnitude of current increment of load current $\Delta I_{L}$ and the magnitude of current increment of fault current $\Delta I_{F}$ as shown in (2).

$$
\Delta I_{L}<\Delta I<\Delta I_{F}
$$

Fault selective relay and distance relay $\Delta \mathrm{I}$ may malfunction due to the inrush current of transformer during unloading or overcurrent when a sudden load increases. To relieve this issue, the protection relay needs to block the low harmonics, this is because the low order harmonics in fault current is relatively less than the load current [7].

In using distance relaying, the ratio between $\mathrm{V}$ and $\mathrm{I}$ could be too similar for high impedance fault and heavy load cases. It will be difficult to find out that there is a 
fault. For the heavy load case, in case if you switch in a resistive load, together with the previous load, there should be difference in the phase in the before and after switch in. However for high impedance fault situation, the angle between $\mathrm{V}$ and $\mathrm{I}$ for each phase will be different. This is different from resistive load switching to the three phases. The angle will be the same.

In practice, relay must detect sensitive fault protection while operating in heavily loaded circuits. Heavy loading prevents adequate sensitive setting of the phase overcurrent elements to detect lower magnitude phase-to-ground faults, residual ground overcurrent elements are available to provide sensitive ground fault protection without tripping under balanced heavy load conditions [2].

HIFs on electrical power networks involve arcing and/or nonlinear characteristics of fault impedance which cause cyclical pattern and distortion. The objective of most detection schemes is to recognize the unique features in patterns of the currents and voltages associated with HIFs. Typically, identification techniques involve two basic steps: pattern recognition and feature extraction. Pattern recognition methods intend to detect characteristic voltage and current distortions caused by the faults [8].

This paper aims to show from the first principle that by using phasor difference, it is possible to show the different between high impedance faults from heavy load cases. In some situations when the high impedance faults and heavy load are so similar as such there is hardly any difference in the voltage and current waveforms, however, it is also possible to separate high impedance fault from heavy load cases with wavelet analysis based on the patterns of transient effect. Further details will be demonstrated later.

\section{WAVELET ANALYSIS}

\section{A. Applications of Wavelet Analysis}

Transient Detection: Wavelet is a good technique for detecting sudden changes, increasing and decreasing trends of data and defining the role of factors with small and large scale effects [9].

Data filtering: There are applications in which wavelet is used for data filtering. For example, it integrated with computational intelligent techniques, such as Artificial Neural Network for power system load forecasting. Neural network affects significantly with bad quality training data, it will deceive the network and in some cases it can't produce a meaningful solution. It needs robust data filtering. Wavelet transform is used to smooth the training data, which are used to train the artificial neural network (ANN) to forecast the load, solar radiation and wind speed [10].

\section{B. Discrete Wavelet Analysis}

The Discrete Wavelet Transform (DWT) is a powerful mathematic tool for time-frequency analysis of nonstationary signals. It uses multi-resolution filter banks for the signal analysis. The general form of DWT at L-level is written in terms of $\mathrm{L}$ detail coefficients $d_{j}(k)$, and the Lth Level approximation coefficients $\mathrm{c}_{\mathrm{L}}(\mathrm{k})$ can be expressed as in [11].

$$
x(t)=\sum_{j=1}^{L} \sum_{k} d_{j}(k) \psi_{j}(t)+\sum_{k} c_{L}(k) \varphi_{L}(t)
$$

Where, the functions $\varphi_{L}(t)$ and $\psi_{j}(t)$ are known as the scaling function and the mother wavelet respectively.

After each level of decomposition, the input data is branched into two outputs, one associated to the upper halfband of the input signal and the other one to the lower halfband. In each branch, the sampling rate is half of that of the input data to that branch. Therefore, the overall sampling rate remains constant. Since decomposition of the DWT proceeds only on one branch, the lower half-band branch, it will result with different information.

For many signals, the low-frequency content carries the most important information about the signal. In general, the low-frequency content carries the signal identity. The highfrequency content, on the other hand, gives details of the signal.

The approximation will be the high scale or lowfrequency components of a signal. The details are the lowscale, high frequency components.

A single level decomposition puts a signal through 2 complementary low-pass and high-pass filters as given in the equations below:

$$
\begin{aligned}
& y_{\text {low }}[n]=\sum_{k=-\infty}^{\infty} h_{0} x[k][2 n-k] \\
& y_{\text {high }}[n]=\sum_{k=-\infty}^{\infty} h_{1} x[k][2 n-k]
\end{aligned}
$$

where $h_{0}$ is the low-pass filter and $h_{1}$ is the high-pass filter [12].

\section{CASE STUdy}

PowerFactory was used to perform the case studies for the HIF and heavy loading in a distribution network. For the convenience of the readers to demonstrate the basic issue \& concept and reduce the complexity of the problem. The following parameters were used: Generator: 125 MVA, $22 \mathrm{kV}$, Power factor 0.85 , Transformer: $22 / 11 \mathrm{kV}$, $125 \mathrm{MVA}$. It is assumed that it is an ideal transformer. 


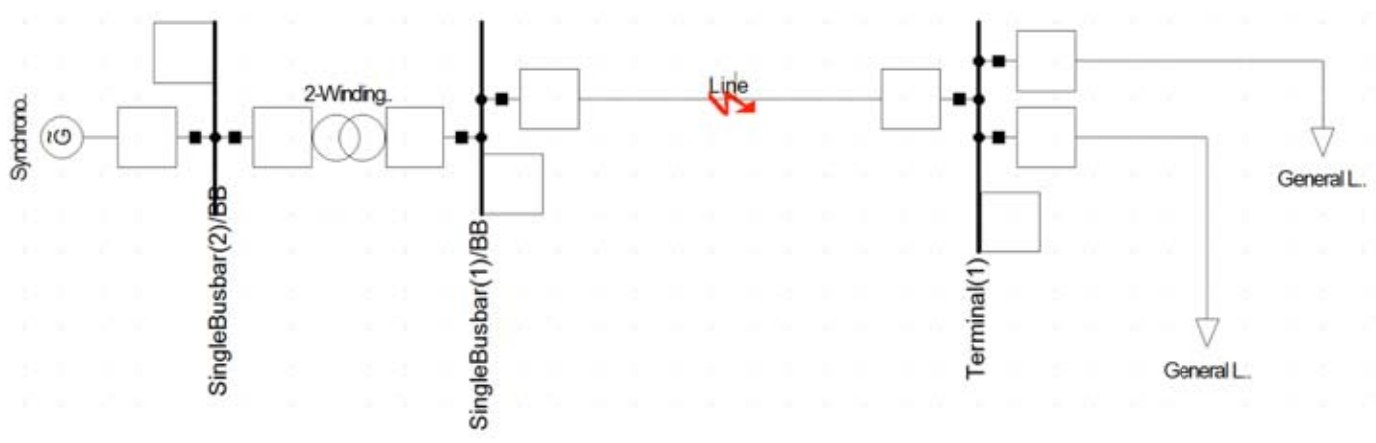

Figure 1: Network model with a 1-phase short circuit on distribution line.

Table 1: Distribution line parameters

\begin{tabular}{|l|l|}
\hline Rated Current & $0.963 \mathrm{kA}$ \\
\hline Positive Sequence Impedance, Z1 & $0.1181563 \Omega$ \\
\hline Positive Sequence Impedance, Angle & $79.66383^{\circ}$ \\
\hline Positive Sequence Resistance, R1 & $0.0212 \Omega$ \\
\hline Positive Sequence Reactance, X1 & $0.1162389 \Omega$ \\
\hline Zero Sequence Resistance, R0 & $0.0848 \Omega$ \\
\hline Zero Sequence Reactance, X0 & $0.4649556 \Omega$ \\
\hline
\end{tabular}

Discrete Wavelet Transform was computed with MATLAB, with the mother wavelet $\mathrm{db} 20$ and at Level 5.

\section{A. High Impedance Fault}

\section{1) Resistive Fault Case}

In this case study, the fault impedance is purely resistive, with the following conditions:

1-phase short circuit on the line at $0.02 \mathrm{~s}$

Fault resistance $=230 \Omega$

Waveform has the same current amplitude as that for the heavy load case. Figures 2 and 3 show the results for the voltage and current waveforms and the decomposed signals from the wavelet analysis.

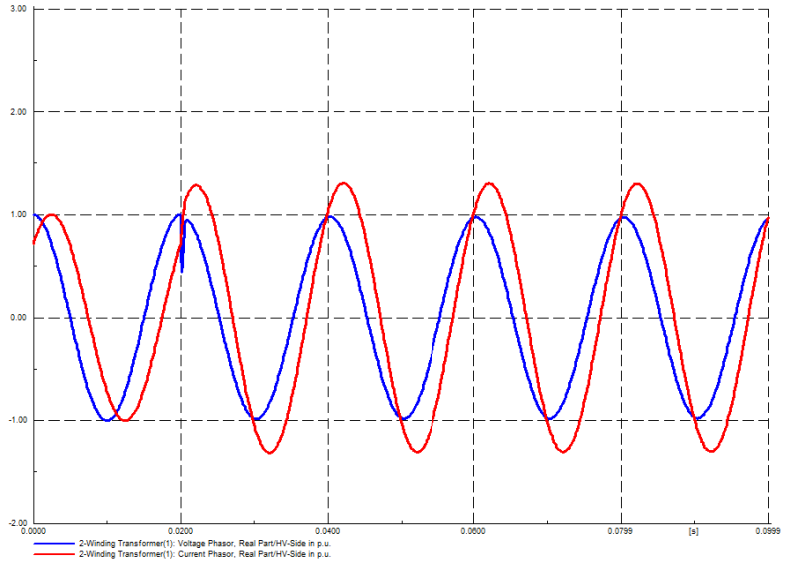

Figure 2: Voltage (blue) and Current (red) waveforms for

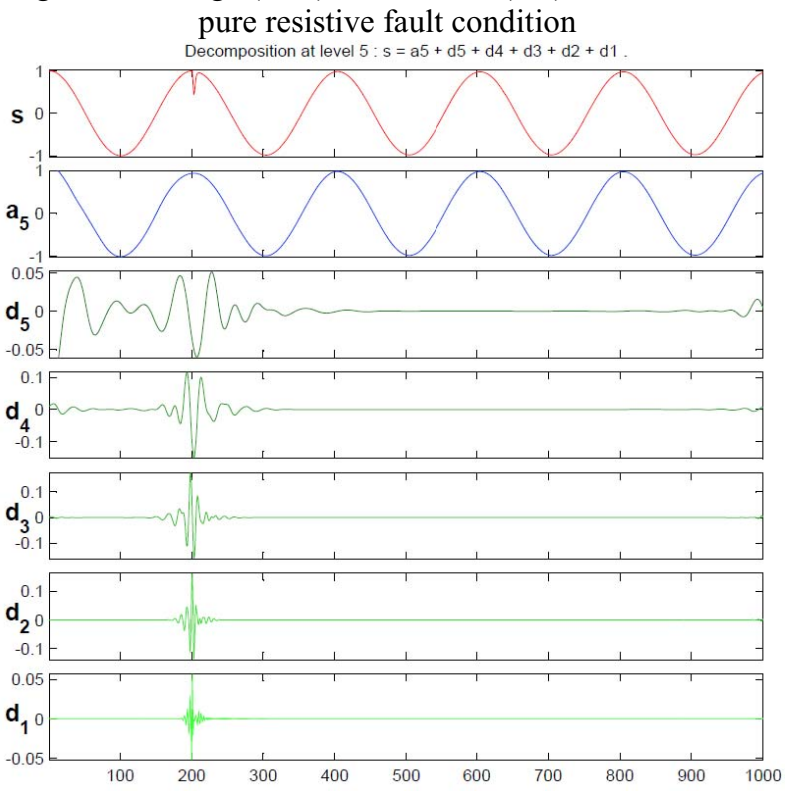

Figure 3: Discrete Wavelet Transform of Voltage in Fig. 2 


\section{2) Resistive and Reactive Fault Case}

In this case study, the fault impedance has a combination of resistive and reactive components, with the following conditions:

1-phase short circuit on the line at $0.02 \mathrm{~s}$

Fault resistance $=168 \Omega$

Fault reactance $=168 \Omega$

Figures 4 and 5 show the results for the voltage and current waveforms and the decomposed signals from the wavelet analysis.

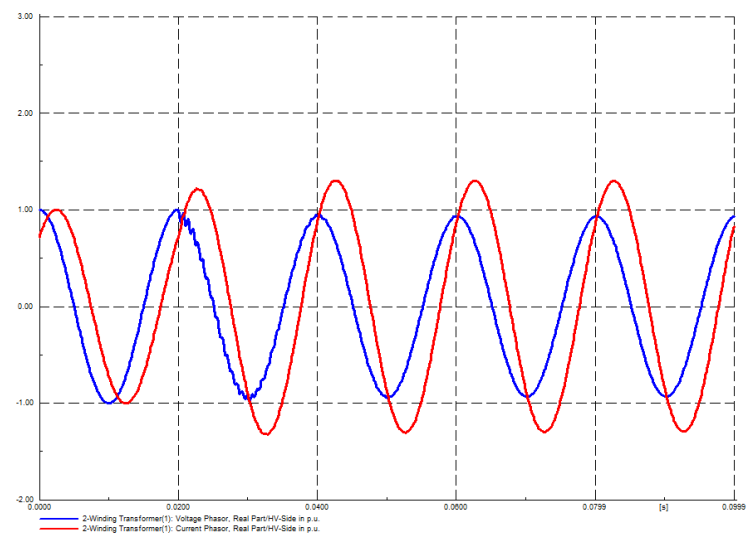

Figure 4: Voltage (blue) and Current (red) waveforms with resistive and reactive fault condition

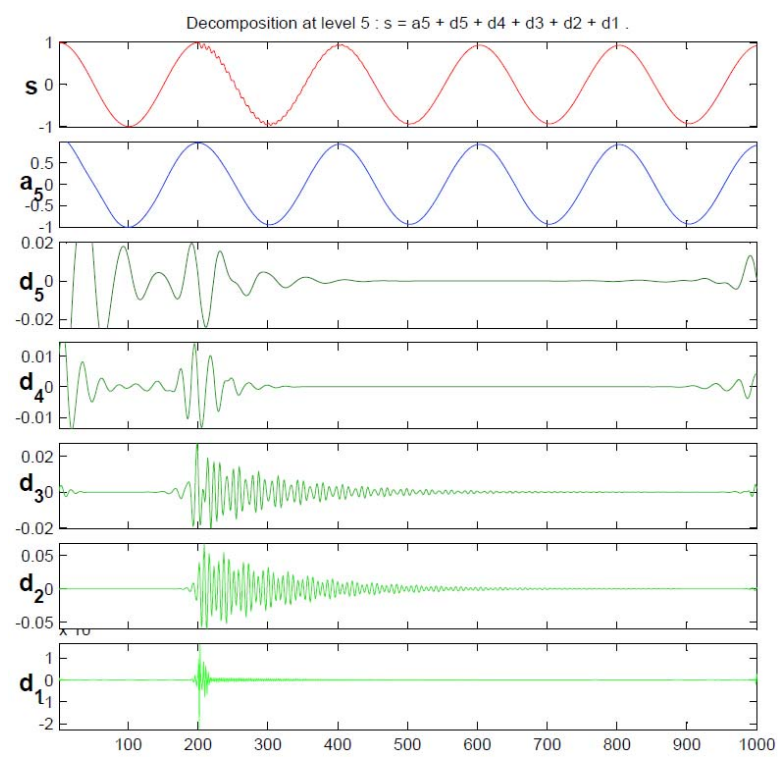

Figure 5: Discrete Wavelet Transform of Voltage in Fig. 4

\section{3) Reactive Fault Case}

In this case study, the fault impedance is purely reactive, with the following conditions:

1-phase short circuit on the line at $0.02 \mathrm{~s}$

Fault reactance $=160 \Omega$

Phase difference after fault is $0.003 \mathrm{sec}$, which is 54 degrees.

Figures 6 and 7 show the results for the voltage and current waveforms and the decomposed signals from the wavelet analysis.

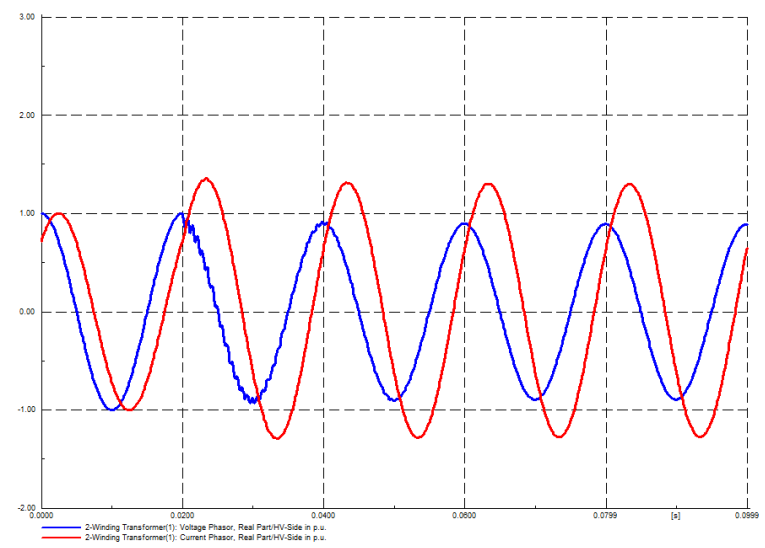

Figure 6: Voltage (blue) and Current (red) waveforms for pure reactive fault condition
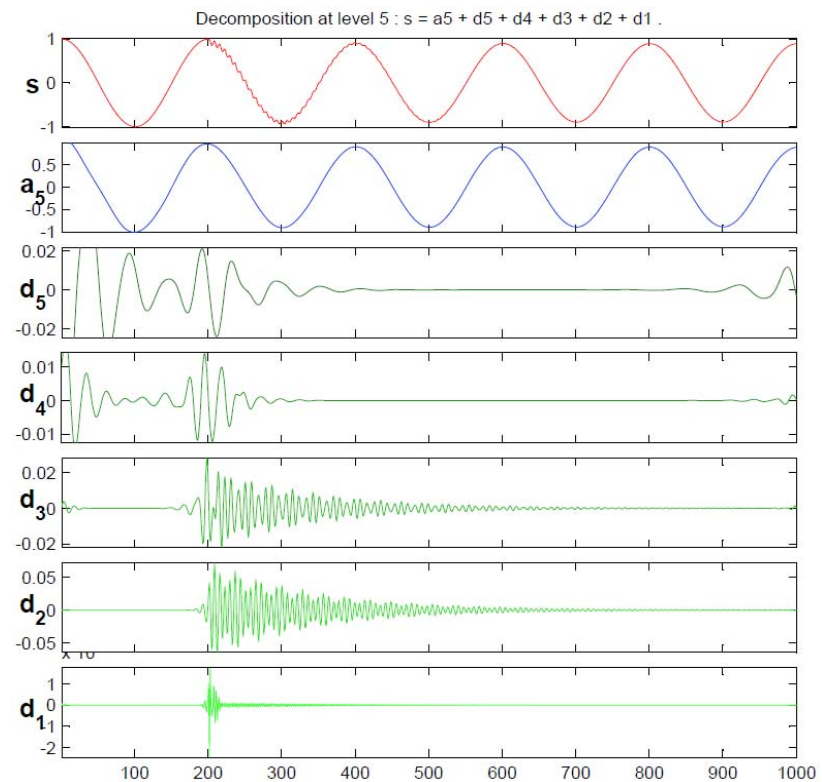

Figure 7: Discrete Wavelet Transform of Voltage in Fig. 6 


\section{B. Heavy Load}

In this case study, additional load is added to the busbar via 'short circuit' at $0.02 \mathrm{~s}$ with the following conditions:

General Load: 90MW, 84MVAr

Additional Load: 52MW

Figures 8 and 9 show the results for the voltage and current waveforms and the decomposed signals from the wavelet analysis.

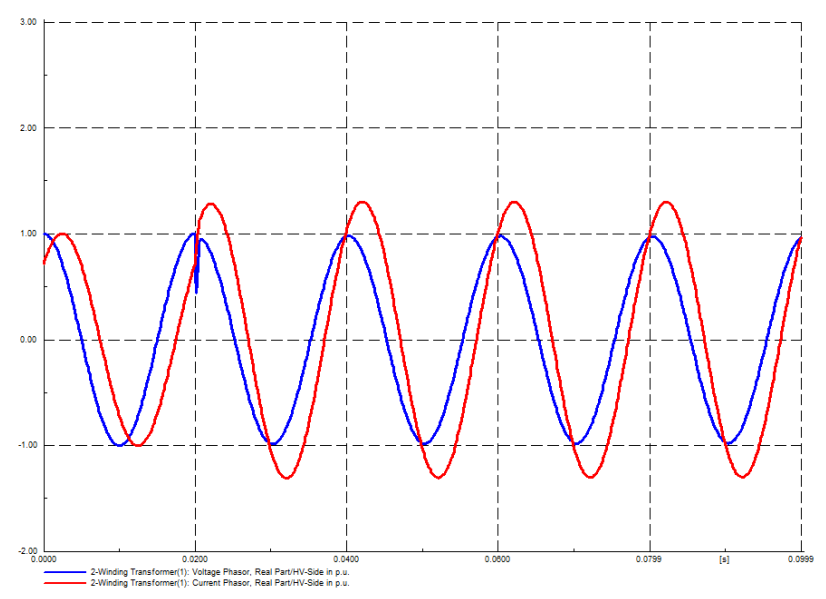

Figure 8: Voltage (blue) and Current (red) waveforms for the heavy load condition
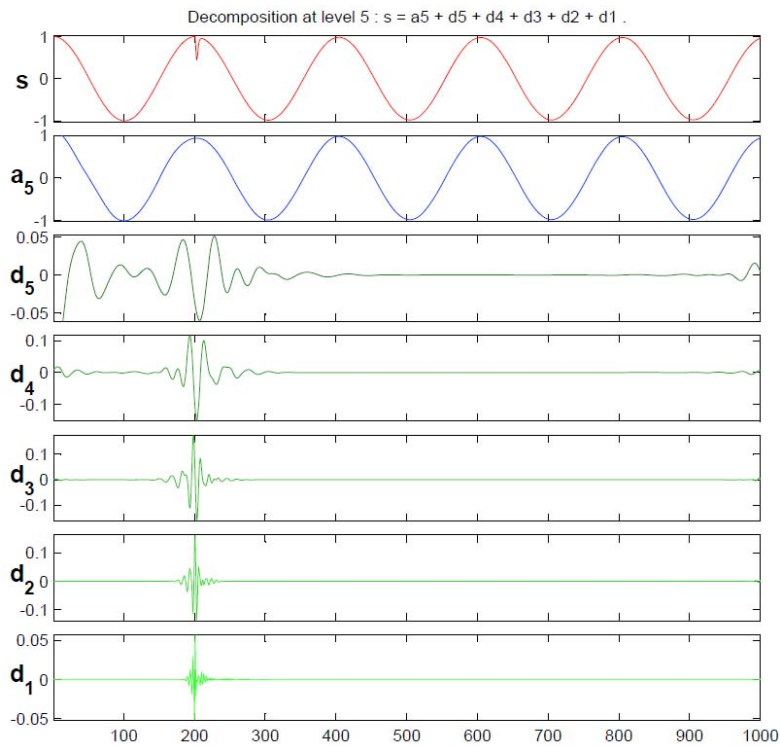

Figure 9: Discrete Wavelet Transform of Voltage in Fig. 8

Before HIF or load switching the phasor angle between current and voltage is $36^{\circ}$. Table 2 below shows the phase angle after the events.
Table 2: Phase angle difference for different faults

\begin{tabular}{|c|c|c|}
\hline Conditions & $\begin{array}{c}\text { Phase angle } \\
\text { in degree }\end{array}$ & $\begin{array}{c}\text { Phase angle } \\
\text { difference after } \\
\text { and before fault }\end{array}$ \\
\hline $\begin{array}{c}\text { Resistive + Reactive } \\
\text { Fault }\end{array}$ & 54 & 18 \\
\hline Resistive Fault & 36 & 0 \\
\hline Reactive Fault & 54 & 18 \\
\hline $\begin{array}{c}\text { Heavy Load (Resistive } \\
\text { Type) }\end{array}$ & 36 & 0 \\
\hline
\end{tabular}

\section{Big Data Analytics in Power System}

The purpose of information processing is convert data to form the information, which could allocate the training patterns such as fault types and fault signature into clusters by a grouping technique. Then the clusters are assigned to classes, which are the expected fault events or heavy load in the power system. The number of clusters is increased and their positions are updated automatically during learning.

Large amounts of data from intelligent sensors on the operation of the grid infrastructure which is managed in a systematic manner [4].

From GTM Research, it reported that the value of the global utility data analytics market is at a cumulative of $\$ 20$ billion between 2013 and 2020. In the technical level, it is essential for scattered and incompatible data sources to be integrated to avoid the garbage problem. More data enables more robust statistical analysis and give better outcome based on data mining analysis to allow more accuracy and flexible control. For example smart meter data may enhance demand response.

Presently, it is estimated that the automated metering infrastructure (AMI) annual rate of data intake is $30 \mathrm{~TB}$ while that of smart homes is $700 \mathrm{~TB}$. With this volume of data, advanced analytics are required to support complex decision-making. In power system protection as studied in this paper, due to the fact that there are infinite combinations of cases so that the amount of data generated will be so significant and also the amount of time which is available to extract useful information from them could be so limited; this is a real challenge.

It is vital for all the sensor devices or measurement units are in a good condition and functioning well to measure and transmit voltage and current signals to main substation. Algorithms exist for identifying HIFs and for locating faults through detailed analysis of monitored data, but the quality of available measurements on distribution circuits is often insufficient to assist them [13].

Protection needs to be responded in milliseconds. Complex relationship between data needs to be extracted therefore power system fault analytics are required. Data needs to be processed fast with high accurate information to minimize risk under an environment of high 
uncertainties. Needlessness to say, late decision leads to missing opportunities. The present big data technology will have a huge potential application to reduce this problem.

\section{DISCUSSUION}

It can be seen that some simulation results are very similar and cannot be picked up by wavelet. In the heavy load case, as the MW load was connected to the system, this is equivalent to a resistive fault. Hence the signals from wavelet analysis are very similar.

In resistive case, the voltage can change sharply, but with the inclusion of inductance, the voltage charge is very much smaller and this is due to the effect of induced voltage from the inductor.

While for the impedance fault, there are many more ripples and they are due to inductive energy circulation. Since there is a resistive component, so there is a decrease in ripple magnitude as time goes by. Table 3 summarizes the finding.

Table 3: Summary of simulation results on phase angle difference for different scenarios

\begin{tabular}{|l|l|l|l|l|}
\hline Fault & Load & $\begin{array}{l}\text { Phase angle } \\
\text { between V/I } \\
\text { before fault }\end{array}$ & $\begin{array}{l}\text { Phase angle } \\
\text { between V/I } \\
\text { after fault }\end{array}$ & $\begin{array}{l}\text { Can wavelet detect } \\
\text { switching event (fault or } \\
\text { load switching) }\end{array}$ \\
\hline Resistance & Resistance & Zero & Zero & No \\
\hline Resistance & Impedance & Much larger & Near zero & No \\
\hline Impedance & Resistance & Zero & Much larger & Yes \\
\hline Impedance & Impedance & $\begin{array}{l}\text { Much larger } \\
\text { than zero }\end{array}$ & $\begin{array}{l}\text { Much larger } \\
\text { than zero }\end{array}$ & Yes \\
\hline
\end{tabular}

\section{CONCLUSIONS}

A re-visit to high impedance fault and heavy load classifications has been carried out. This paper has a tutorial value in this aspect. In addition to this, the author pointed out that under the so called big data context, there could be cases where high impedance faults and heavy load could not be separated with the present technology and techniques with phase measurements and wavelet analysis.

In real-life situations; the signal may be so small that the present technology may not be able to have devices sensitive enough to provide a reliable result. As imagination has no limit, many emerging new techniques are coming out in the big data analytics and it is foreseen that by digging further different features between high impedance faults and heavy load, it is believed that with the integration of new features extraction methods, power protection could have significant improvements to increase reliability and reduce outages of a power network. Scientists and engineers would need to work together to produce a workable solution.

\section{REFERENCES}

[1] Carl L. Benner and B. Don Russell, "Practical highimpedance fault detection on distribution detection", IEEE Transactions on Industry Applications, Vol. 33, No. 3, May/June 1997, 635-640.
[2] SEL-751 Feeder Protection Relay, Directional Overcurrent, Arc-Flash Detection, and High-Impedance Fault Detection, SEL-751 Data Sheet, Schweitzer Engineering Laboratories, Inc., 2011.

[3] Alexandra von Meier, David Culler and Alex McEachern, "Micro-synchrophasors for distribution systems", IEEE ISGT, 2013.

[4] Mladen Kezunovic, Le Xie and Santiago Grijalva, "The role of big data in improving power system operation and protection", 2013 IREP Symposium-Bulk Power System Dynamics and Control -IX (IREP), August 25-30, 2013.

[5] Marek Michalik, Waldemar Rebizant, Miroslaw Lukowicz, Seung-Jae Lee and Sang-Hee Kang, "High-impedance fault detection in distribution networks with use of wavelet-based algorithm", IEEE Transactions on Power Delivery, Vol. 21, October 2006.

[6] Chul-Hwan Kim, Hyun Kim, Young-Hun Ko, Sung-Hyun Byun, Raj K. Aggarwal and Allan T. Johns, "A novel faultdetection technique of high-impedance arcing faults in transmission lines using the wavelet transform", IEEE Transactions on Power Delivery, Vol. 17, No. 4, October 2002, 921-929.

[7] F. B. Costa, B. A. Souza, N. S. D. Brito, J. A. C. B. Silva and W. C. Santos, "Real-time detection of transients induced by high impedance faults based on the boundary wavelet Ttransform", IEEE Transactions on Industry Applications, DOI 10.1109/TIA.2015.2434993.

[8] T. M. Lai, L. A. Snider, E. Lo, and D. Sutanto, "Highimpedance fault detection using discrete wavelet transform and frequency range and RMS conversion", IEEE Transactions on Power Delivery, Vol. 20, No. 1, Jan 2005, 397-407.

[9] Diao-yan Hua, Li-chun Ming and Shang Yan, "Waveletbased fault diagnosis scheme for power system relay protection", Proceedings of the Seventh International Conference on Machine Learning and Cybernetics, IEEE Kunming China, July 2008, 2187-2191.

[10] Ahmed T. Ghareeb and Osama A. Mohammed, "Waveletadaptive ANN forecaster for renewable energy sources for continuous supply in microgrid applications", IEEE PES General Meeting, 2013.

[11] F. B. Costa, "Boundary wavelet coefficients for real-time detection of transients induced by faults and power quality disturbances," IEEE Transactions on Power Delivery, Vol. 29, No. 6, 2674-2687, Dec. 2014.

[12] Stephane G. Mallat, "A theory for multiresolution signal decomposition: the wavelet representation", IEEE Transactions on Pattern Analysis and Machine Intelligence, 1989.

[13] A. H. A. Bakar, M. S. Ali, ChiaKwang Tan, H. Mokhlis, H. Arof and H. A. Illias, "High impedance fault location in 11 $\mathrm{kV}$ underground distribution systems using wavelet transforms", Electrical Power and Energy Systems, Elsevier, 2014. 